\title{
A Molecular Link Between Interleukin 22 and Intestinal Mucosal Wound Healing
}

\author{
Xiaoyan Sun, ${ }^{1-4}$ Laura Chalmers, ${ }^{1-3}$ Xiaobing Fu, ${ }^{4, *}$ and Min Zhao ${ }^{1-3, * *}$ \\ ${ }^{1}$ Departments of Dermatology and ${ }^{2}$ Ophthalmology, and ${ }^{3}$ Institute for Regenerative Cures, University of California Davis \\ School of Medicine, Sacramento, California. \\ ${ }^{4}$ Wound Healing and Cell Biology Laboratory, Institute of Basic Medical Science, Trauma Center of Postgraduate Medical \\ School, Chinese PLA General Hospital, Beijing, People's Republic of China.
}

Background: Interleukin 22 (IL-22) and signal transducer and activator of transcription 3 (STAT3) are two important regulators of inflammation. Crohn's disease and ulcerative colitis are considered inflammatory bowel diseases (IBDs), due to the belief that these diseases result from dysregulated responses of the intestinal immune system to bacteria present in the commensal flora.

The Problem: It is debated whether a breakdown of immune tolerance is the primary cause of these diseases or occurs downstream of an initial defect of the intestinal barrier and intestinal epithelial cells (IECs).

Basic/Clinical Science Advances: Recent reports suggest a crucial role for IL22 in the regulation of gut inflammation as well as epithelial barrier integrity. Local $I L-22$ gene delivery enhances expression of its downstream effector, STAT3, within colonic epithelial cells and induces both STAT3-dependent expression of mucus-associated molecules and restitution of mucus-producing goblet cells. IEC-specific deletion of STAT3 results in significant susceptibility to experimental colitis with a striking defect in epithelial restitution. STAT3 activation, thus, may regulate immune homeostasis in the gut by promoting IL-22-dependent mucosal wound healing.

Clinical Care Relevance: The importance of IL-22/STAT3 signaling in IEC wound healing suggests a critical role for epithelial homeostasis in IBDs.

Conclusion: Effective healing of the IECs could be considered a primary target in the development of treatments for IBDs. IL-22/STAT3 signaling exerts a protective role in the process of intestinal mucosal wound healing and may thereby provide a promising therapeutic approach to the treatment of IBDs.

\section{BACKGROUND}

THE INTESTINAL EPITHELIUM provides the first line of immune defense against pathogens and a mechanism for processing food antigens. Defective healing of mucosa underlies many important intestinal diseases, including inflammatory bowel diseases (IBDs) such as Crohn's disease (CD) and ulcerative colitis. It is generally believed that disregulation of the intestinal immune response and disruption of the intestinal epithelial barrier are key features of IBDs. The respective contributions of the immunological pathways and/or the intestinal mucosal wound healing response have not yet been fully elucidated.

Recent reports have identified a crucial role for interleukin 22 (IL-22) in the regulation of both gut inflammation and epithelial barrier integrity. $I L-22$ gene delivery leads to rapid amelioration of dextran sodium sulfate (DSS)-induced colitis, ${ }^{1}$

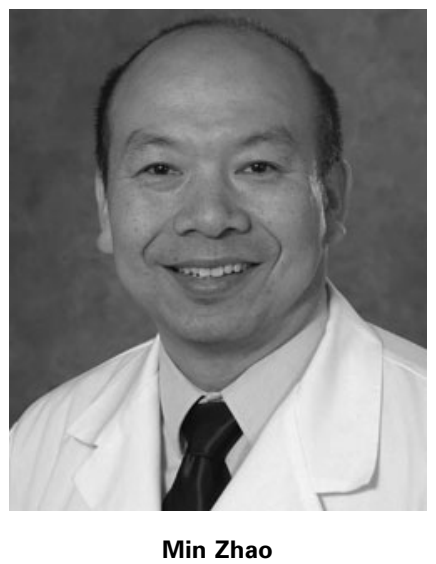

Submitted for publication August 8, 2011. *Correspondence: Wound Healing and Cell Biology Laboratory, Institute of Basic Medical Science, Trauma Center of Postgraduate Medical School, Chinese PLA General Hospital, 28 Fu Xing Road, Beijing 100853, People's Republic of China (e-mail: fuxiaobing@vip.sina.com).

** Correspondence: Institute for Regenerative Cures, University of California Davis School of Medicine, 2921 Stockton Blvd., Room 1617, Sacramento, CA 95817 (e-mail: minzhao@ucdavis .edu).
Abbreviations and Acronyms
$C D=$ Crohn's disease
CECs $=$ colonic epithelial cells DSS $=$ dextran sodium sulfate $\mathrm{IL}=$ interleukin
IBDs = inflammatory bowel diseases
IECs = intestinal epithelial cells JAK kinases $=$ Janus kinases $\mathrm{NK}=$ natural killer
STAT3 $=$ signal transducer and activator of transcription 3 


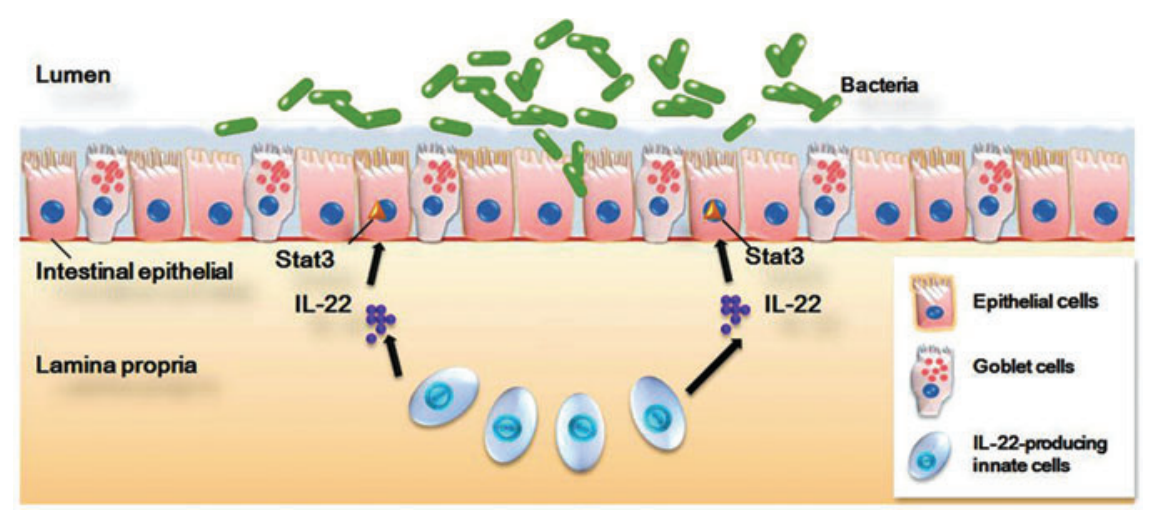

Figure 1. The mucosal defense system: the intestinal epithelium lines the mucosal surface of the intestinal tract and is a key player in intestinal homeostasis. It provides the first line of immune defense against pathogens and a mechanism for proper processing of food antigens. Recent reports have identified a crucial role for IL-22/STAT3 signaling in the regulation of both gut inflammation and epithelial barrier integrity, which may provide a promising therapeutic approach to the treatment of IBDs, as well as other intestinal disorders (redrawn from Fig. 4 in Ref. ${ }^{22}$ ). IL, interleukin; STAT3, signal transducer and activator of transcription 3. Color images available online at www.liebertpub.com/wound

\section{TARGET ARTICLES}

1. Pickert G, Neufert C, Leppkes M, Zheng Y, Wittkopf N, Warntjen M, et al.: STAT3 links IL22 signaling in intestinal epithelial cells to mucosal wound healing. J Exp Med 2009; 206: 1465.

2. Neufert C, Pickert G, Zheng Y, Wittkopf N, Warntjen M, Nikolaev A, Ouyang W, Neurath $\mathrm{MF}$, and Becker C: Activation of epithelial STAT3 regulates intestinal homeostasis. Cell Cycle 2010; 9: 652.

whereas $I L-22$ knockout ( $I L-22^{-/-}$) mice show increased intestinal epithelial damage, along with systemic bacterial burden and significantly increased mortality. ${ }^{2}$ Sugimoto et al. demonstrated that $I L-22$ local gene delivery enhanced signal transducer and activator of transcription 3 (STAT3) activation within colonic epithelial cells (CECs), and induced both STAT3-dependent expression of mucus-associated molecules and restitution of mucus-producing goblet cells. ${ }^{1}$ Pickert et al. showed that intestinal epithelial STAT3 activation regulated immune homeostasis in the intestine by promoting IL-22-dependent mucosal wound healing. ${ }^{3}$ IL-22 and STAT3 are two important mediators of intestinal mucosal inflammation and wound healing (Fig. 1), and as such, we will focus on the roles of these two important immune signaling molecules in mucosal epithelial wound healing.

\section{CLINICAL PROBLEM ADDRESSED}

IBDs such as CD and ulcerative colitis are considered two of the most prevalent gastrointestinal diseases, and are lifelong diseases occurring early in life. The incidence of IBDs has markedly increased in recent years. The cellular/molecular mechanisms of IBDs have not yet been clarified, and immunosuppressive agents that nonspecifically reduce inflammation and immunity have been used as the mainstay treatments for IBDs. Evidence indicates that a dysregulation of mucosal immunity in the gut of IBD causes an overproduction of inflammatory cytokines and trafficking of effector leukocytes into the bowel, thus leading to an uncontrolled intestinal inflammation. ${ }^{4}$ Whether a breakdown of immune tolerance is the primary cause of these diseases or occurs downstream of an initial defect in the IECs/intestinal epithelial barrier is not yet clear.

\section{RELEVANT BASIC SCIENCE CONTEXT}

Recent research in the field of IBD has led to the identification of several cytokine pathways that may be potential therapeutic targets. One of these pathways comprises IL-22, a less well-defined cytokine associated with mucosal immune disorders. IL-22 was initially known as IL-10-related T-cellderived inducible factor. ${ }^{5}$ Studies have revealed that IL-22 targets cells of the digestive, skin, and respiratory organ systems, thereby mediating epithelial innate immunity. ${ }^{6-8}$ IL-22 is produced by a subgroup of IL-17-producing CD4 ${ }^{+}$T-cells (Th17 cells). ${ }^{8,9}$ More recently, a unique IL-22 producing $\mathrm{CD}^{+}{ }^{+} \mathrm{T}$ helper subset (Th22) has been identified in 
psoriatic skin samples. ${ }^{7}$ Human Langerhans cells may differentiate $\mathrm{T}$ cells into the IL-22-producing $\mathrm{T}$ helper phenotype. ${ }^{10}$ Innate human immune cell types such as natural killer (NK) cells and lymphoid tissue inducer cells also produce IL-22. ${ }^{11,12} \mathrm{~A}$ distinct NK cell population $\left(\mathrm{NKp} 46^{+}\right)$present in the mucosa-associated lymphoid tissue produces high levels of IL-22 and may be the main source of IL-22 in mucosal immunity. ${ }^{13}$ IL-22 can target epithelial cells instead of immune cells. ${ }^{9,14,15}$ IL-22, therefore, unlike other cytokines, has functions in epithelial cells as well as in inflammation.

The recent discovery that IL-22 activates STAT3 has improved our understanding of how IL-22 regulates intestinal epithelial wound healing in addition to its important role in the immune response. STAT3 has been identified as the key molecule involved in mediating the wound healing effect of IL-22. STAT3 is a transcription factor activated by a variety of cytokines and growth factors. On activation by Janus kinases (JAK kinases), STAT3 translocates to the nucleus, where it regulates expression of genes involved in apoptosis, proliferation, migration, and survival, depending on the cell type. It has been shown that high levels of phosphorylated STAT3, the active form, are present in both CD and ulcerative colitis, but not in healthy intestinal tissue. ${ }^{16}$

\section{EXPERIMENTAL MODEL OR MATERIAL: ADVANTAGES AND LIMITATIONS}

Experimental IBD was induced in the murine model in these studies. This is a well-used animal model for IBDs. The advantage of using this model is the highly detailed knowledge of its genetic makeup. Deletion of STAT3 specifically in IECs has several advantages. Pickert et al. demonstrated the effects of STAT3 in epithelial cells, and excluded possible confounding involvement of the effects of STAT3 in immune cells. After removal of the colitis inducer DSS, wild-type animals with STAT3 gene intact in intestinal epithelium recovered, whereas animals with STAT3 gene knockout in IECs showed significantly delayed epithelial wound healing, epithelial erosion, and weight loss. These results clearly demonstrate the role of IL-22/ STAT3 in IECs. IL-22 could promote wound healing through increased cell migration in IECs in wound assays. ${ }^{6}$ The protective role of IL-22 in IBD is likely to be linked to its functions in enhancing epithelial innate defense mechanisms and epithelial barrier integrity. IBDs result from complicated pathological processes, which include inflammatory responses, breaking down of the epithelial barrier integrity, and it is possible that these two pathological processes may interact with and exacerbate each other. An initial breakdown of epithelial barrier integrity may severely impact the immune response. The contribution of each of these processes, however, is yet to be defined.

\section{DISCUSSION OF FINDINGS AND RELEVANT LITERATURE}

IL-22 signals through a class II cytokine receptor composed of two subunits, IL-22R1 and IL-10R2. ${ }^{9}$ IL-10R2 is widely expressed in immune cells (T, B, and NK cells), whereas IL-22R1 expression is present in a variety of nonimmune tissues, including skin, lung, small intestine, liver, colon, kidney, and pancreas. $^{8,9}$ On binding to its heterodimeric receptor complex, IL-22R1 and IL-10R2, IL-22 activates the JAK1 and Tyk2 tyrosine kinases, thus leading to the phosphorylation of STAT3 and, to a lesser extent, of STAT1 and STAT5.,

T cell-specific STAT3 knockout mice showed impaired T cell proliferation. ${ }^{17}$ Activation of the $I L-6 /$ $S T A T 3$ cascade in lamina propria $\mathrm{T}$ cells induced prolonged survival of pathogenic $\mathrm{T}$ cells and, indeed, inactivation of this cascade contributed to the attenuation of chronic intestinal inflammation. ${ }^{18}$ Disruption of innate immune responses may be brought about either by genetically engineered inactivation of the STAT3 cascade in the CECs or by macrophage-induced intestinal inflammation. ${ }^{19}$ These results were confirmed in a subsequent study by using a conditional STAT3 knockout mouse strain in which efficient deletion of STAT3 protein in innate cells (macrophages and CECs), but not acquired cells ( $\mathrm{T}$ and $\mathrm{B}$ cells), caused development of a lethal colitis immediately in adults. ${ }^{20}$

A novel microinjection-based local gene-delivery system allowed the targeting of inflamed mucosa and supplementation of local IL-22 expression restricted to the delivered site. ${ }^{1}$ This method demonstrated the role of IL-22-mediated activation of the innate immune pathway in Th2-mediated colitis: $I L-22$ gene delivery enhanced STAT3 activation specifically within CECs and induced both STAT3dependent expression of mucus-associated molecules and restitution of mucus-producing goblet cells. ${ }^{1}$ Importantly, IL-22 contributed to the rapid amelioration of local intestinal inflammation by enhancing the production of membrane-bound mucins (Muc-1, $-3,-10$, and -13). ${ }^{1}$ Membrane-bound mucins are stored in goblet cell vacuoles and form a static external barrier at the epithelial surface. Recent findings have indicated that Muc- 2 and Muc-3 contribute to the suppression of experimental colitis. It is, 


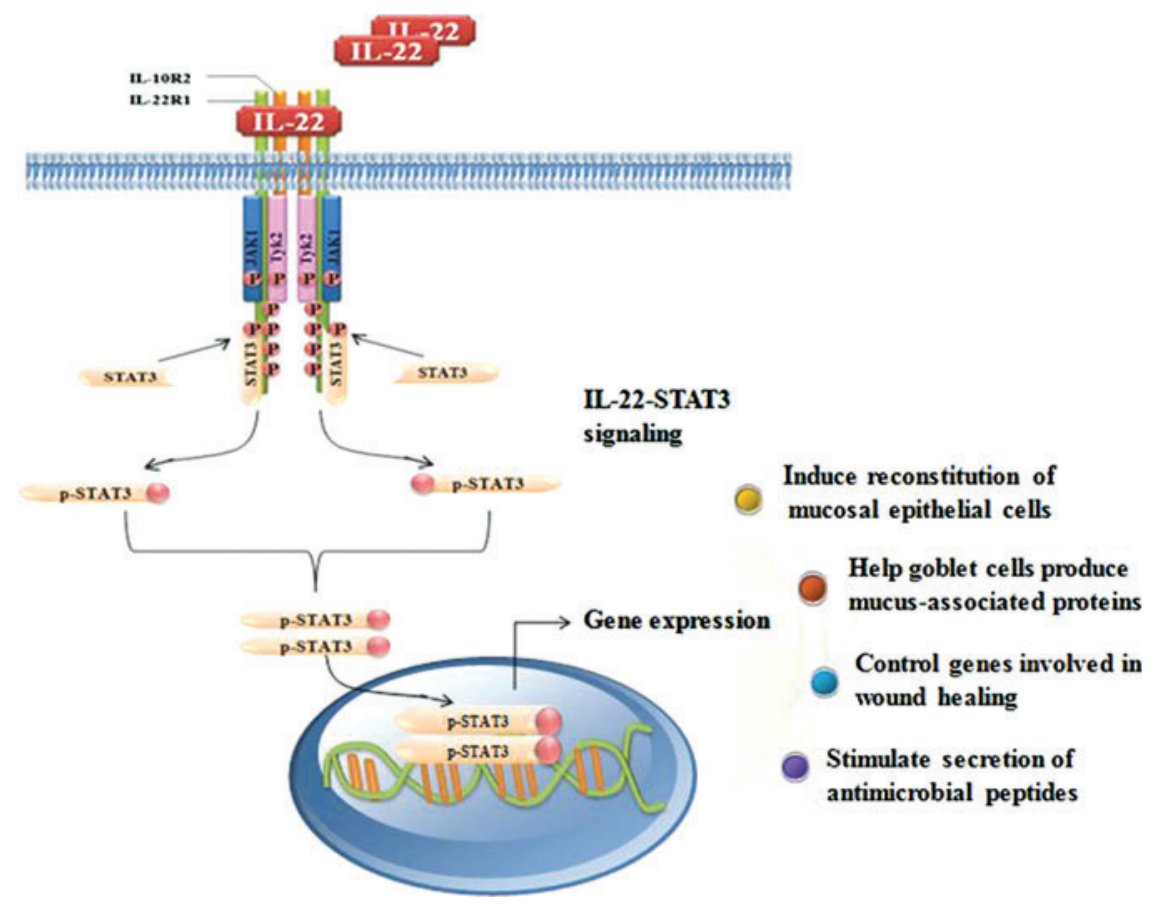

Figure 2. Schematic model of the regulation of intestinal homeostasis by the IL-22/STAT3 pathway in IECs. In the intestine, both innate and adaptive immune cells are capable of producing IL-22 in response to various stimuli. For instance, NKp46 + cells present in the mucosa-associated lymphoid tissue are the major innate source of IL-22. On binding to its heterodimeric receptor complex, IL-22R1 and IL-10R2, IL-22 activates the JAK1 and Tyk2 tyrosine kinases, thus leading to the phosphorylation of STAT3. The active STAT3 then translocates to the nucleus, where it regulates gene expression, which subsequently contributes to the suppression of intestinal inflammation, induces the expression of mucus-associated proteins and the restitution of mucus-producing goblet cells, and enhances the motility of IECs in the process of mucosal wound healing. NK, natural killer; JAK kinases, Janus kinases; IECs, intestinal epithelial cells. Color images available online at www.liebertpub.com/wound

therefore, possible that IL-22 stimulates mucus production and goblet cell restitution under intestinal inflammatory conditions and also contributes to the rapid attenuation of this inflammation. Consistent with these findings, Pickert et al. demonstrated a pivotal role for STAT3 activation in IECs for the restoration of the balance at the mucosal surface of the intestine in an experimental colitis model. ${ }^{3}$ By using genetically engineered mice with intestinal epithelial STAT3 deletion, Pickert et al. defined IECs as important players in STAT3-dependent intestinal homeostasis and indicated that epithelial STAT3 activation in DSS-induced colitis was dependent on IL-22, which was secreted by CD11 ${ }^{+}$cells in response to Toll-like receptor stimulation. ${ }^{3}$ Activation of epithelial STAT3 regulated genes associated with cellular stress, apoptosis, and wound repair, thus revealing an important role of STAT3 in resolution of inflammation in the gut. ${ }^{3}$ To further test the role of IL-22 and STAT3 signaling in human intestine, Neufert and colleagues extended their recent findings by showing that activation of STAT3 also occurs in human IECs from patients with IBD. ${ }^{21}$ The authors stained colonic biopsies obtained from patients with CD with an antibody specific to phospho-STAT3, and found that samples from patients with active CD showed elevated STAT3 activity in the lamina propria as well as in IECs compared with controls. ${ }^{21}$ This result confirmed their findings using experimental colitis in mice in human tissues. ${ }^{3}$ Although the repetitive injection of a specific anti-IL-22 antibody every other day had little impact on the development of experimental colitis, antibody-mediated neutralization of IL-22 resulted in a significant delay in weight gain during the resolution phase, thus suggesting a protective role for IL-22 during resolution of experimental colitis. ${ }^{21}$

The protective roles of IL-22/STAT3 in intestinal mucosal host defense may include the following: (1) IL-22 induces the proliferation and reconstitution of mucosal epithelial cells through the activation of STAT3, which enhances the healing response and prevents further penetration of microbes into the epithelial layer $^{9}$; (2) IL-22 helps goblet cells to regenerate and produce mucus-associated proteins, which form the essential static external barrier separating intestinal flora from IECs ${ }^{9}$; (3) STAT3 activation in IECs after induction of colitis or injury regulates the signaling pathways that are commonly involved in wound healing and which subsequently induce cell proliferation, remodeling of extracellular matrix and cell migration, and 
contribute to the process of mucosal wound healing; and (4) IL-22 stimulates the production and secretion of various antimicrobial peptides, such as defensins, cathelicidins, and C-type lectins, from IECs and Paneth cells. ${ }^{9}$ These antimicrobial peptides further sequester and directly kill the invading pathogens through various mechanisms (Fig. 2).

\section{INNOVATION}

One innovative aspect of the target articles is to unmask the molecular crosstalk between the immune system and the intestinal epithelium. Based on the experimental data, the authors hypothesize a model in which a barrier defect within the intestinal epithelium facilitates the translocation of intestinal microbes into the submucosa where they encounter dendritic cells and other innate immune cells. ${ }^{1,3,21}$ On pattern recognition including activation of Toll-like receptors by innate immune cells, early defense mechanisms are activated that include the production of IL-22 by IL-22-producing innate cells (e.g., CD11c ${ }^{+}$cells). Subsequently, IL-22 binds to the IL-22 receptor complex expressed by IECs that initiates a signaling cascade and results in the activation of STAT3 in IECs. STAT3 phosphorylation in IECs then promotes a transcriptional cascade which comprises the induction of targets that favor mucosal wound healing. ${ }^{1,3,21} \mathrm{Sev}$ eral mechanisms including proliferation, antiapoptosis, and production of antimicrobial proteins such as $\operatorname{RegIII} \beta$ and RegIII $\gamma$ are thereby involved in restoration of intestinal homeostasis. Thus, targeting the IL-22/STAT3 signaling pathway in IECs is a promising therapeutic approach in situations where intestinal homeostasis is compromised, for example, CD, and ulcerative colitis.

\section{TAKE-HOME MESSAGE}

\section{Basic science advances}

IL-22 is a central regulator of mucosal host defense, and is crucial for epithelial barrier integrity. IL-22 binds to its receptor complex, thereby resulting in the activation of STAT3 in IECs. Activation of STAT3 significantly enhances wound healing responses in IECs through the upregulation of many genes important to epithelial wound healing, stimulation of IEC proliferation and migration, reduction of epithelial erosion in vivo, and subsequent recovery of body weight after DSS-induced colitis in mice. The mucosal healing responses also include expression of mucus-associated proteins, the restitution of mucusproducing goblet cells, and enhancement of IEC motility. Recovery of the barrier should also contribute to the suppression of intestinal inflammation. Thus, the protective role of IL-22/STAT3 in intestinal mucosal host defense may include (1) inducing the proliferation and restitution of mucosal epithelial cells through the activation of STAT3; (2) helping goblet cell regeneration and production of mucus-associated proteins; (3) modulating the genes that are commonly involved in wound healing and induce cell proliferation, remodeling of extracellular matrix, and cell migration during mucosal wound healing; and (4) stimulating secretion of antimicrobial peptides. The IL-22/STAT3 signaling involved in intestinal homeostasis sheds new light on the molecular mechanisms underlying the mucosal wound healing process.

\section{Clinical science advances}

Animal experiments have identified the molecular basis of IL-22/STAT3 signaling in IBDs, and found similarities to pathogenic functions in chronic inflammatory skin diseases. In IBDs, IL-22/STAT3 signaling plays an essential role in epithelial wound healing. Further investigation of the mechanisms of IL22/STAT3 signaling in psoriasis and IBDs will yield significant insights into epithelial wound healing and inflammatory responses. Elucidating these conflicting effects will aid in the design of therapeutic treatments targeting the IL22/STAT3 pathway for the treatment of IBDs.

\section{Relevance to clinical care}

Importantly, healing of the epithelium and recovery of the barrier function has significant effects in improving the overall health of the organism, including mucosal erosion and body weight recovery. These are important parameters that clinicians aim at improving. Healing of the epithelium may have an equally important role in the management of IBDs.

However, the protective role of IL-22/STAT3 signaling in IBD marks an important first step in linking the immune system to the intestinal epithelium. Future studies should focus on defining the context-dependent functions of IL22/STAT3 signaling in intestinal inflammation and repair, including the effect of microbial communities at epithelial barrier surfaces, and the spatial and temporal co-expression of other proinflammatory or regulatory cytokines. In addition, the role of IL-22/STAT3 signaling during tumorigenesis should be addressed, to develop therapeutic approaches that will target intestinal mucosal wound healing while avoiding tumor development or exacerbation.

\section{CAUTION, CRITICAL REMARKS, AND RECOMMENDATIONS}

Recent human- and experimental animal-based studies have highlighted the IL-22/STAT3 signaling pathway in IBDs. The activation of IL-22/ STAT3 has a protective role in the intestinal epithelium through which cells of the innate immune responses regulate host defense, inflammation, and tissue homeostasis at epithelial barrier surfaces. Accordingly, targeting the IL-22/STAT3 signaling pathway may be a promising therapeutic approach for patients with intestinal disorders such as CD and ulcerative colitis.

Development, plasticity, and cell-lineage relationships of innate sources of IL-22 in mouse and human diseases remain unclear. Further examination 
pin-pointing the role of different IL-22-expressing subsets is needed to underline the regulatory network of cytokine expression in immunity and homeostasis of the intestinal tract. In addition, the IL-22/STAT3 signaling pathway plays a protective role in the regulation of intestinal inflammation, enhances wound repair, and is crucial for epithelial barrier integrity. Unfortunately, similar to many cytokines that hold dual roles, IL-22 plays an essential role in the pathogenesis of psoriasis. ${ }^{7,9,14}$ IL-22 can synergize with other proinflammatory cytokines to induce many of the pathogenic phenotypes of keratinocytes and exacerbate disease progression. ${ }^{7,9,14}$ Further investigation aimed at elucidating these apparently conflicting effects will aid in the design of therapeutic treatments targeting the IL-22/STAT3 pathway for the treatment of persistent infections, chronic inflammation, and autoimmune diseases. Finally, current studies have revealed the roles of IL-22/STAT3 signaling in aiding tissue repair. ${ }^{1,3,14,21,22}$ In particular, IL-22 is constitutively expressed by $\mathrm{CD}_{11 \mathrm{c}^{+}}$cells within the small intestine, a tissue that undergoes constant renewal and is susceptible to tumorigenesis. It is, therefore, critical to devise an elaborate regulatory method to keep IL22/STAT3 signaling under control to promote mucosal wound healing during inflammation while avoiding tumorigenesis, because STAT3 activation may be related to tumor formation.

\section{FUTURE DEVELOPMENT OF INTEREST}

Future studies will focus on defining the contextdependent functions of IL-22/STAT3 signaling in tissue inflammation and repair, including the effect of microbial communities at the epithelial barrier surface and the spatial and temporal coexpression of other proinflammatory or regulatory cytokines. In addition to the role of IL-22/ STAT3 signaling under inflammatory conditions, the role of this signaling pathway in tumorigenesis needs to be more closely examined. Better understanding is important for development of therapeutic treatments targeting the IL-22/ STAT3 pathway for the treatment of intestinal mucosal disorders, which block potential tumorigenic side-effects during the mucosal woundhealing process, but leave the protective functions of IL-22 intact.

\section{ACKNOWLEDGMENTS AND FUNDING SOURCES}

Research in the author's lab is supported by grants from NIH (1R01EY019101), California Institute of Regenerative Medicine (RB1-01417), NSF (MCB-0951199), Research to Prevent Blindness, Inc., NSFC (30901564, 30730090, 81121004), and the National Basic Science and Development Programme (973 Programme, 2012CB518105). The author (MZ) served as an ad hoc consultant for Johnson \& Johnson.

\section{AUTHOR DISCLOSURE AND GHOSTWRITING}

The authors declare no conflict of interest. This article was not written by any writer other than the authors.

\section{REFERENCES}

1. Sugimoto K, Ogawa A, Mizoguchi E, Shimomura Y, Andoh A, Bhan AK, Blumberg RS, Xavier RJ, and Mizoguchi A: IL-22 ameliorates intestinal inflammation in a mouse model of ulcerative colitis J Clin Invest 2008; 118: 534.

2. Zheng Y, Valdez PA, Danilenko DM, Hu Y, Sa SM, Gong Q, Abbas AR, Modrusan Z, Ghilardi N, de Sauvage FJ, and Ouyang W: Interleukin-22 mediates early host defense against attaching and effacing bacterial pathogens. Nat Med 2008; 14: 282.

3. Pickert G, Neufert C, Leppkes M, Zheng Y, Wittkopf $N$, Warntjen M, Lehr HA, Hirth S, Weigmann B, Wirtz S, Ouyang W, Neurath MF, and Becker C: STAT3 links IL-22 signaling in intestinal epithelia cells to mucosal wound healing. J Exp Med 2009; 206: 1465.
4. Strober W, Fuss I, and Mannon P: The fundamental basis of inflammatory bowel disease. J Clin Invest 2007; 117: 514.

5. Dumoutier L, Louahed J, and Renauld JC: Cloning and characterization of $\mathrm{IL}-10$-related $\mathrm{T}$ cellderived inducible factor (IL-TIF), a novel cytokine structurally related to IL-10 and inducible by IL-9. J Immunol 2000; 164: 1814

6. Aujla SJ and Kolls JK: IL-22: a critical mediator in mucosal host defense. J Mol Med 2009; 87: 451.

7. Eyerich S, Eyerich K, Pennino D, Carbone $T$, Nasorri F, Pallotta S, Cianfarani F, Odorisio T, TraidlHoffmann C, Behrendt H, Durham SR, SchmidtWeber CB, and Cavani A: Th22 cells represent a distinct human $T$ cell subset involved in epidermal immunity and remodeling. J Clin Invest 2009; 119: 3573.
8. Wolk K, Witte E, Witte K, Warszawska K, and Sabat R: Biology of interleukin-22. Semin Immunopathol 2010; 32: 17.

9. Ouyang W: Distinct roles of IL-22 in human psoriasis and inflammatory bowel disease. Cytokine Growth Factor Rev 2010; 21: 435

10. Fujita H, Nograles KE, Kikuchi T, Gonzalez J, Carucci $\mathrm{JA}$, and Krueger JG: Human Langerhans cells induce distinct IL-22-producing CD4 + T cells lacking IL-17 production. Proc Natl Acad Sci USA 2009; 106: 21795

11. Colonna M: Interleukin-22-producing natural killer cells and lymphoid tissue inducer-like cells in mucosal immunity. Immunity 2009; 31: 15

12. Spits $\mathrm{H}$ and Di Santo JP: The expanding family of innate lymphoid cells: regulators and effectors of 
immunity and tissue remodeling. Nat Immunol 2011; 12: 21.

13. Cella M, Fuchs A, Vermi W, Facchetti F, Otero K, Lennerz JK, Doherty JM, Mills JC, and Colonna M: A human natural killer cell subset provides an innate source of IL-22 for mucosal immunity. Nature 2009; 457: 722.

14. Boniface K, Bernard FX, Garcia M, Gurney AL, Lecron JC, and Morel F: IL-22 inhibits epidermal differentiation and induces proinflammatory gene expression and migration of human keratinocytes. J Immunol 2005; 174: 3695.

15. Sa SM, Valdez PA, Wu J, Jung K, Zhong F, Hall L, Kasman I, Winer J, Modrusan Z, Danilenko DM, and Ouyang W: The effects of IL-20 subfamily cytokines on reconstituted human epidermis suggest potential roles in cutaneous innate defense and pathogenic adaptive immunity in psoriasis. J Immunol 2007; 178: 2229.
16. Lovato P, Brender C, Agnholt J, Kelsen J, Kaltoft K, Svejgaard A, Eriksen KW, Woetmann A, and Ødum N: Constitutive STAT3 activation in intestinal T cells from patients with Crohn's disease. J Biol Chem 2003; 278: 16777.

17. Takeda K, Kaisho T, Yoshida N, Takeda J, Kishimoto $T$, and Akira S: Stat3 activation is responsible for IL-6-dependent $T$ cell proliferation through preventing apoptosis: generation and characterization of T cell-specific Stat3-deficient mice. J Immunol 1998; 161: 4652.

18. Atreya R, Mudter J, Finotto S, Müllberg J, Jostock T, Wirtz S, Schütz M, Bartsch B, Holtmann M, Becker C, Strand D, Czaja J, Schlaak JF, Lehr HA, Autschbach F, Schürmann G, Nishimoto N, Yoshizaki K, Ito H, Kishimoto T, Galle PR, Rose-John S, and Neurath MF: Blockade of interleukin 6 trans-signaling suppresses T-cell resistance against apoptosis in chronic intestinal inflammation: evidence in Crohn's disease and experimental colitis in vivo. Nat Med 2000; 6: 583.
19. Welte T, Zhang SS, Wang T, Zhang Z, Hesslein DG, Yin Z, Kano A, Iwamoto Y, Li E, Craft JE, Bothwell AL, Fikrig E, Koni PA, Flavell RA, and Fu XY: STAT3 deletion during hematopoiesis causes Crohn's disease-like pathogenesis and lethality: a critical role of STAT3 in innate immunity. Proc Natl Acad Sci USA 2003; 100: 1879.

20. Alonzi T, Newton IP, Bryce PJ, Di Carlo E, Lattanzio G, Tripodi M, Musiani P, and Poli V: Induced somatic inactivation of STAT3 in mice triggers the development of a fulminant form of enterocolitis. Cytokine 2004; 26: 45.

21. Neufert C, Pickert G, Zheng Y, Wittkopf N, Warntjen $M$, Nikolaev $A$, Ouyang $W$, Neurath $M F$, and Becker C: Activation of epithelial STAT3 regulates intestinal homeostasis. Cell Cycle 2010; 9: 652.

22. Li Z, Burns AR, Byeseda Miller $S$, and Smith CW: CCL20, \{gamma\}\{delta\} T cells, and IL-22 in corneal epithelial healing. FASEB J 2011; 25: 2659. 